\title{
ENERGIRA: EL JUEGO INFANTIL QUE GENERA ENERGÍA A TRAVÉS DE LA ROTACIÓN
}

\author{
ENERGIRA: THE CHILDREN'S GAME \\ THAT GENERATES ENERGY \\ THROUGH ROTATION
}

ALEJANDRA REYNAFARJE VIDAL

Universidad de Lima

Ante la inminente necesidad de incorporar el componente de sostenibilidad en el ámbito de la arquitectura, el diseño de proyectos sostenibles de menor escala es igual de importante que aquellos a nivel urbano para generar un cambio desde grupos pequeños de personas y comunidades, ya que todos somos beneficiarios de una mejora en nuestra huella ecológica. El presente artículo muestra a Energira: un juego infantil capaz de iluminar su entorno generando energía a través del movimiento de rotación. Este fue diseñado y construido por un grupo de estudiantes de arquitectura y donado a una casa hogar en San Juan de Lurigancho.

juego infantil, energía, dínamo, sostenibilidad
Recibido: 30 de junio del 2020

Aprobado: 3 de noviembre del 2020

doi: https://doi.org/10.26439/limaq2021.n008.5558

With the imminent need to incorporate sustainability to the field of architecture, the design of small-scale sustainable projects are just as important as those at the urban level to bring about change in groups of people and communities, since we would all benefit from a positive change in our ecological footprint over time. This article presents Energira: que merry-goround children's game capable of illuminating its surroundings by generating energy though the movement of rotation. This was designed and built by a group of architecture students and donated to a girls' home in San Juan de Lurigancho.

merry-go-round, children's game, energy, dynamo, sustainability 


\section{INTRODUCCIÓN}

Si bien la sostenibilidad era vista como un componente opcional o adicional, está claro que para generaciones futuras de arquitectos se ha vuelto una necesidad. El construir con materiales de ciclos cerrados, mantener un uso responsable del agua, incorporar energías limpias y cuidar la huella ecológica deben poder normalizarse para asegurar un buen desarrollo para las siguientes generaciones.

En el ámbito de la arquitectura, la sostenibilidad suele pensarse como una oportunidad para proyectos de grandes escalas, ya sean obras de vivienda, espacios públicos, culturales e incluso a escala urbana. Sin embargo, para abordar un cambio tan significativo, se debe poder incorporar la sostenibilidad no solo a grandes obras, sino también a proyectos de menor escala. Además, es importante reconocer la necesidad de empezar tomando acciones pequeñas, dado que los beneficios de cambiar nuestros estilos de vida serían parte de la solución a un problema de todos nosotros.

Los conceptos de sostenibilidad y desarrollo sostenible se volvieron conocidos a raíz del documento Nuestro futuro común, el informe de la Comisión Mundial sobre el Medio Ambiente publicado en 1987 y liderado por Gro Harlem Brundtland, la entonces primera ministra de Noruega. En el documento se define sostenible como "aquel desarrollo que satisface las necesidades del presente sin comprometer la capacidad de las generaciones futuras para satisfacer sus propias necesidades" (Organización de las Naciones Unidas, 1987, p. 23).

Si traducimos esta definición a una menor escala, se puede tomar la idea de algo sostenible como un elemento que puede mantenerse por sí mismo, es decir, que sea autosuficiente. El proyecto que decidimos diseñar englobando esta nueva idea de sostenibilidad fue Energira: un juego infantil que es capaz de generar energía limpia a través del movimiento.

\section{PRIMERAS INTENCIONES}

Si bien el encargo inicial no especificaba el uso del proyecto, desde un inicio se supo que el enfoque principal sería incentivar a un grupo de niños a moverse y generar energía de esta manera, por lo que optamos por un juego infantil. En este caso, decidimos trabajar con la generación de energía a través del movimiento, es decir, poder transformar energía cinética en electricidad a través de la rotación.

Inicialmente, se pensó en una solución formal similar a la de un columpio, dado que es un juego infantil utilizado universalmente. La funcionalidad estaría clara, y la idea era generar energía a través del movimiento pendular (mientras el columpio se balancea hacia adelante y atrás). Sin embargo, "en la práctica el péndulo pierde siempre una parte de la energía mecánica y depende de su coeficiente aerodinámico" (Escholarium, s. f., párr. 3). Esta combinación de factores causa no solo que haya pérdida de energía, manifestada en forma de energía térmica, sino también dificulta el poder predecir un patrón de 
movimiento, y, por ende, la cantidad de energía cinética que puede generar. Por otro lado, la energía del movimiento rotacional es más estable y predecible.

Se dice que un objeto que gira en torno a un eje tiene energía cinética de rotación [...] La energía cinética por rotación es [...] dada por la expresión $\frac{1}{2} I \omega^{2}$, donde $I$ es el momento de inercia del objeto y $\omega$ es su velocidad angular. (Giancoli, 2009, p. 210)

Es por esta razón que optamos por cambiar a un diseño que nos permitiera generar y conservar energía a través de la rotación constante. De esta manera, se minimizaría la pérdida de energía cinética, y se facilitaría la conversión de energía cinética a energía eléctrica para finalmente lograr cargar una batería e iluminar los alrededores.

El diseño debía ser adaptable para cualquier entorno y ser capaz de transformar la energía en electricidad o conservar esta energía generada a través del movimiento.

\section{PROCESO DE CONSTRUCCIÓN}

El nuevo diseño se inspiró en los juegos de merry-go-round o carrusel infantil, los cuales consisten en dos planchas circulares superpuestas, de manera que una se mantiene estática en el suelo y la otra logra girar en su mismo eje. El juego tendría, además, una tira de luces LED ubicada en su circunferencia. De esta manera, la energía generada por los niños al dar vueltas sería transformada a energía eléctrica. Con suficientes rotaciones, el Energira sería capaz de alumbrar su entorno de manera autosuficiente, a través del movimiento.

Las dos bases circulares fueron cortadas de una plancha de triplay de $1,22 \mathrm{~m}$ $\times 2,44 \mathrm{~m}$, de las cuales salieron dos círculos con un radio de 1,20 $\mathrm{m}$ cada uno. En la plancha circular que giraría (la superior) se perforó un hueco de 5/8" de diámetro, por el cual pasaría un fierro de las mismas medidas. De esta manera, la plancha lograría girar sobre su mismo eje, rotando a su vez el fierro de acero. Para hacer el juego más llamativo, se pintaron las bases de un color rojo brillante.

Dado que es un juego infantil, se construyeron barandas con listones de pino de 2", considerando una altura cómoda para un niño de una talla promedio de $1,20 \mathrm{~m}$. Estos fueron ubicados sobre la superficie giratoria y anclados al triplay mediante placas de acero en $\mathrm{L}$.

Para poder generar energía a través de la rotación, lo cual aportaría al logro de hacer sostenible este proyecto, utilizamos un dínamo de bicicleta. En este caso, el dínamo actúa como un generador eléctrico, que se puede definir como "una máquina destinada a transformar la energía mecánica en eléctrica" (Carranza y Andres, 2013). El dínamo actuaría como intermediario en la conversión de la energía cinética rotatoria generada por los niños a energía eléctrica, y estaría conectado directamente a una batería de $12 \mathrm{~V}$. De esta manera, la energía limpia puede ser tanto conservada (al cargar la batería en su totalidad) o utilizada para encender las luces LED mientras está en movimiento.

Una dificultad que encontramos en ese momento fue diseñar la manera de hacer girar el dínamo mientras que se girara la plancha circular. Para proteger 
el sistema eléctrico, se construyó una caja de contención situada debajo de la base, hecha con maderas recicladas. Dentro de esta, se ubicó un sistema de dos engranajes diseñados y cortados a láser en MDF de $9 \mathrm{~mm}$ de espesor. El engranaje pequeño estaba unido al eje giratorio (al fierro de acero de 5/8”), y conectaba a un engranaje más grande. Al tener dos engranajes de tamaños variables se logró un mayor número de rotaciones en el dínamo, permitiendo así generar más energía en un menor tiempo de rotación.

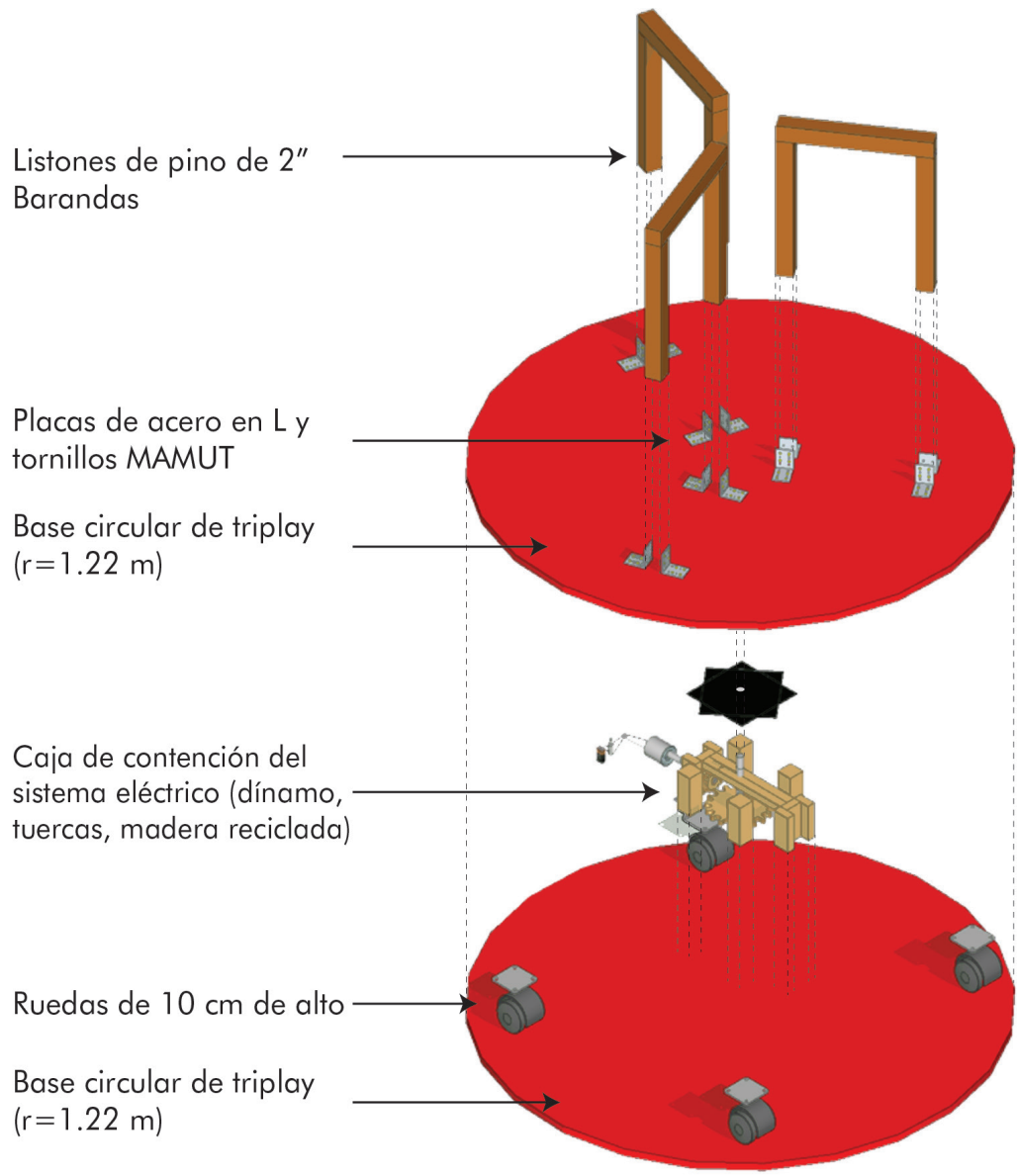

Figura 1. Axonometría explotada del Energira

Elaboración propia
Una vez que el dínamo estuvo bien ubicado y activado, mientras la base giraba sobre su eje, se le conectó una batería de $12 \mathrm{~V}$, la cual alimentaba la tira de luces led pegada en la circunferencia de la base. Luego de que el sistema estuvo completo y funcionando, se le atornillaron cuatro llantas a la base y se perforó el triplay para esconder los cables del circuito y evitar que las llantas pasen por encima, dañándolo a futuro. 
La última dificultad que tuvimos como grupo fue encontrar la manera de asegurar que el fierro del eje principal gire en simultáneo con la base. Para lograr esto, recibimos ayuda del personal de laboratorio de ingeniería de la universidad para soldar una tuerca de 5/8" a dos placas de acero en L; de esta manera, se aseguraría un movimiento de rotación uniforme entre la base, el fierro y, por ende, el dínamo.

En la figura 1 se puede ver una axonometría explotada del diseño final del Energira, con especificaciones de las dimensiones y sus componentes correspondientes.

\section{PRESENTACIÓN DEL PROYECTO}

El proyecto final se presentó en la Universidad de Lima, días antes de ser donado a la casa hogar de niñas en San Juan de Lurigancho.

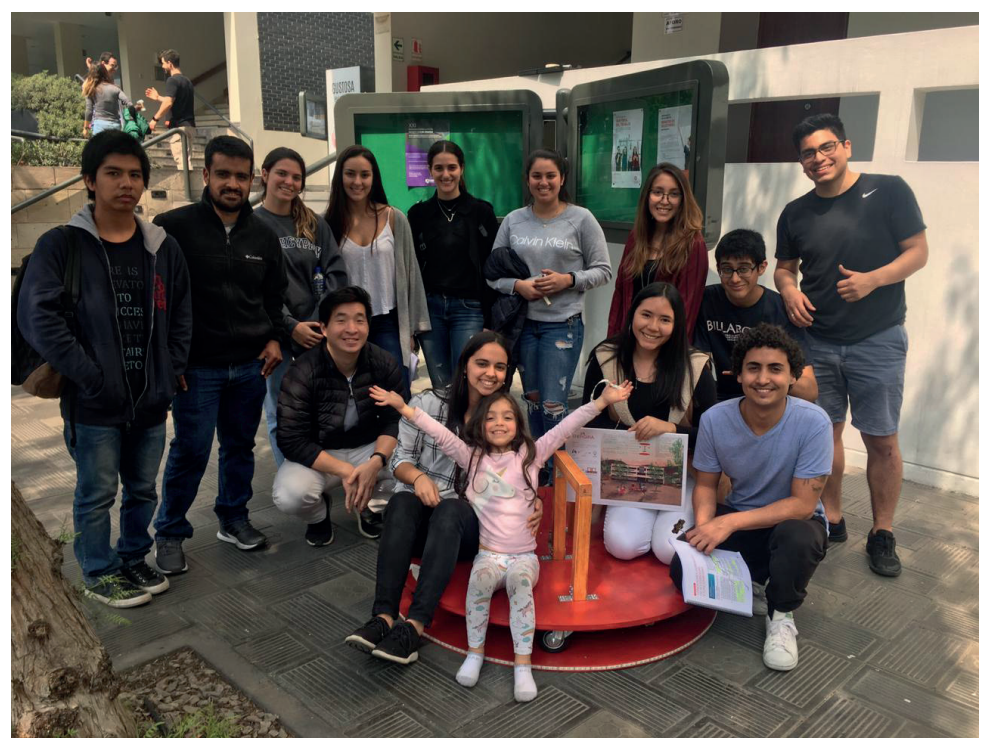

Figura 2

Presentación

final del Energira.

Universidad de

Lima. Integrantes

(de izquierda a

derecha): Leonardo

Gerónimo,

Giovanni Franco,

Araceli Velaochaga,

Alessandra

Rachitoff, Jennifer

Gleiser, Brianna

Jarufe, Brillit

Reyes, Sebastián

Escobedo, Renato

Garavito, Kevin

Fu, Alejandra

Reynafarje, Isabel

Céspedes, Juan

Carlos de las

Casas

Archivo fotográfico

de la autora

\section{DISEÑO FINAL DEL ENERGIRA}

Como complemento al juego infantil, se diseñó un espacio público que pudiera integrar el Energira como elemento sostenible para el parque n. ${ }^{\circ} 1$ en Los Olivos. De esta manera, el juego mostró que puede ser adaptado a un ámbito público, en el cual, si es utilizado frecuente y correctamente, podría generar pequeñas cantidades de energía para abastecer una porción de la iluminación del parque.

Adicionalmente, se incluyeron paneles solares en las luminarias. Si bien un proyecto de poca escala como este no es capaz de generar un cambio notorio en la huella ecológica de un espacio público, una combinación de elementos 
Figura 3. Implementación

del Energira en diseño de espacio

público

Fotografía modelada de Kevin Fu que generen energía limpia puede servir como un punto de partida para un consumo energético más consciente y una mejora en la huella ecológica a futuro.

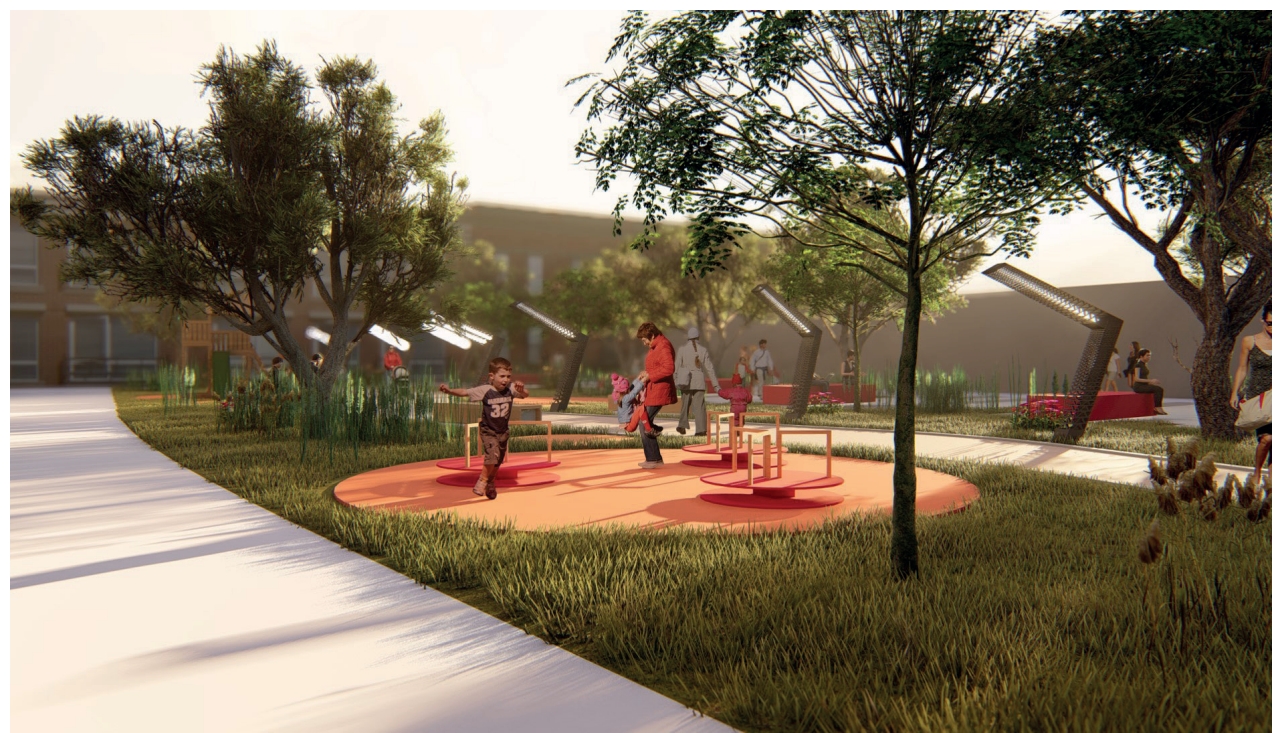

\section{DONACIÓN DEL ENERGIRA}

Una vez terminado el proyecto, el Energira fue donado a una casa hogar de niñas perteneciente a la ONG Hope House Perú. Esta ONG es parte del movimiento sin fines de lucro One for Others, con el objetivo de mejorar las condiciones de vida de niños que viven en pobreza extrema en diferentes áreas del Perú.

Dado que el juego estuvo pensado para poder adaptarse en cualquier entorno, ya sea exterior, interior, público o privado, el lugar al que fue donado el Energira fue producto de una oportunidad, al tener una integrante del grupo con contactos en la ONG mencionada.

Si bien el Energira no fue diseñado pensando específicamente para la situación actual de San Juan de Lurigancho, sirvió como un medio para educar a un grupo pequeño de niñas sobre energía limpia y brindarles un juego nuevo que las incentive a moverse mientras generan su propia electricidad. 

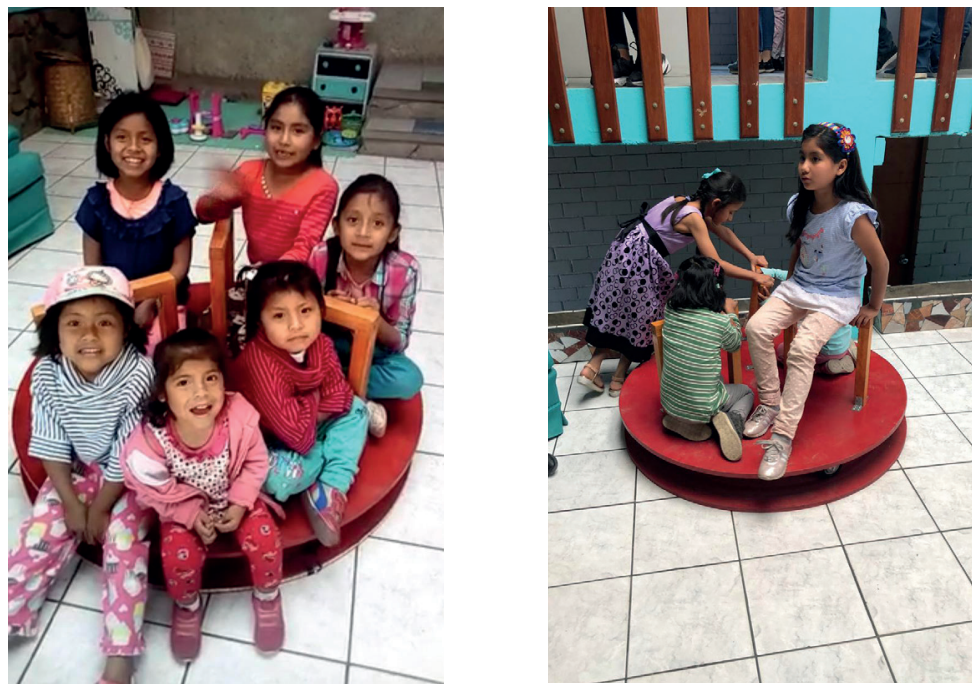

Figura 4.

Donación del Energira. Hope House Perú,

San Juan de Lurigancho

Archivo fotográfico de la autora

\section{CONCLUSIONES Y FUTURAS APLICACIONES}

Se puede decir que logramos construir exitosamente un proyecto sostenible que cumplió su función como juego infantil y como generador de energía limpia, a pesar de las dificultades durante el proceso de construcción. El componente más exitoso del proyecto fue su función como juego infantil, ya que las niñas que lo obtuvieron le dieron uso frecuente en grupos de tres a seis personas y lograron divertirse.

Como diseño de proyecto, sin embargo, hubo algunos aspectos por mejorar a la hora de entregar la versión final del Energira. En el aspecto funcional, el juego puede solo girarse hacia un lado, dado que la composición del dínamo de bicicleta funciona solamente con giros en sentido horario. Esto, en esencia, no es un gran inconveniente, pero es una variable difícil de controlar cuando se trata de niños pequeños dándole uso al proyecto, ya que el juego debe ser manualmente girado por una persona, y girarlo en el sentido contrario podría resultar en problemas en el funcionamiento del dínamo y el circuito de la luz LED.

Por otro lado, la manera en que funciona el sistema energético desde el dínamo hasta la tira de luces LED dificulta tener un entendimiento preciso del aspecto energético. Esto es porque no hay manera concreta de medir cuánta energía produce cada rotación ni cuánto tiempo puede demorar en cargarse la batería de $12 \mathrm{~V}$ por completo.

Más allá de poder hacer una diferencia significativa en la huella ecológica a través de este único proyecto, el Energira busca ayudar a educar a las niñas de esta casa hogar y de la comunidad en San Juan de Lurigancho mediante 
pequeños proyectos como este. Era posible reconocer el impacto que puede causar el educar a pequeñas comunidades sobre la importancia de la energía eléctrica en nuestra vida cotidiana y los beneficios que nos traería el reducir nuestro consumo y aumentar la producción de energía limpia.

Como arquitectos cumplimos una función social, cultural y ambiental tanto en el ámbito público como privado de una sociedad. Por eso, como se ha mencionado anteriormente, adquirimos cierto nivel de responsabilidad a la hora de proponer la manera en que se diseña y se construye a futuro. Dicho esto, la responsabilidad no cae solo en arquitectos y urbanistas, sino también en toda la sociedad. $\mathrm{Y}$ es un proceso lento que se logra a través de pequeños proyectos de impacto positivo en comunidades.

\section{REFERENCIAS}

Giancoli, D. C. (2009). Física 1: principios con aplicaciones. Pearson Education.

Carranza, L., y Andres, W. (2013). Proyecto física eléctrica. Generador de electricidad ecológico. Academia.edu. https://www.academia.edu/33049017/PRO YECTO_FISCA_ELECTRICA_GENERADOR_DE_ELECTRICIDAD_ ECOL $\%$ C3\%93GICO

Escholarium. (s. f.). Péndulo. Energía potencial y energía cinética. https://escho larium.educarex.es/useruploads/r/c/9872/scorm_imported/31224473 588732363393/page_18.htm

Organización de las Naciones Unidas. (4 de noviembre de 1987). Informe de la Comisión Mundial sobre el Medio Ambiente y el Desarrollo. Nota del Secretario General. Ecominga Amazónica. http://www.ecominga.uqam.cal PDF/BIBLIOGRAPHIE/GUIDE_LECTURE_1/CMMAD-Informe-ComisionBrundtland-sobre-Medio-Ambiente-Desarrollo.pdf 